\title{
Quantitative mapping of averaged focal adhesion dynamics in migrating cells by shape normalization
}

\author{
Christoph Möhl*, Norbert Kirchgessner ${ }^{\ddagger \S}$, Claudia Schäfer ${ }^{\star}$, Bernd Hoffmann and Rudolf Merkel \\ Institute of Complex Systems, ICS7: Biomechanics, Forschungszentrum Jülich GmbH, Jülich, Germany \\ *Present address: Image and Data Analysis Facility, DZNE Bonn, Germany \\ ${ }^{\ddagger}$ Present address: Institute of Agricultural Sciences, ETH Zürich, Switzerland \\ $\S$ Author for correspondence (norbert.kirchgessner@argl.ethz.ch) \\ Present address: Department of Medicine, Boston School of Medicine, Boston, MA, USA \\ Accepted 25 July 2011 \\ Journal of Cell Science 125, 155-165 \\ (c) 2012. Published by The Company of Biologists Ltd \\ doi: $10.1242 / j c s .090746$
}

\section{Summary}

The spatially ordered formation and disassembly of focal adhesions is a basic requirement for effective cell locomotion. Because focal adhesions couple the contractile actin-myosin network to the substrate, their distribution determines the pattern of traction forces propelling the cell in a certain direction. In the present study, we quantitatively analyzed the spatial patterning of cell-substrate adhesion in migrating cells by mapping averaged focal adhesion growth dynamics to a standardized cell coordinate system. These maps revealed distinct zones of focal adhesion assembly, disassembly and stability and were strongly interrelated with corresponding actin flow and traction force patterns. Moreover, the mapping technique enables precise detection of even minute responses of adhesion dynamics upon targeted signaling perturbations. For example, the partial inhibition of vinculin phosphorylation was followed by the reduced number of newly formed adhesions, whereas growth dynamics of existing adhesions remained unaffected.

Key words: Actin flow, Cell migration, Focal adhesion dynamics, Traction force

\section{Introduction}

Effective locomotion of migrating cells depends on the coordinated interplay between protrusive, contractile and adhesive components of the cytoskeleton (Lauffenburger and Horwitz, 1996). Traction forces are generated by a viscoelastic network of actin filaments interacting with myosin II motors (Svitkina et al., 1997; Fletcher and Mullins, 2010). These forces are applied to the substrate through distinct focal adhesions (FAs), protein clusters at the cell membrane coupling actin filaments to extracellular matrix proteins (Geiger et al., 2001). Actin filaments and FAs are mainly assembled at the cell front, leading to growth of the actin network and the establishment of a protruding lamella (Anderson and Cross, 2000; Pollard and Borisy, 2003; Zaidel-Bar et al., 2003).

Both components, the actin-myosin network and the FAs, are highly dynamic and influence each other in various ways. Owing to its properties as a polymerizing and contractile gel, the viscous actin network grows at the cell edges and is pulled or pushed towards the cell center by myosin activity or actin polymerization, respectively (Vallotton et al., 2005; Schaub et al., 2007; Gardel et al., 2008; Rubinstein et al., 2009). FAs assembling near the leading edge reduce the rearward movement of actin, by developing a viscous or clutch-like connection to the actin filaments, which generates traction forces on the substrate (Hu et al., 2007; Wang, 2007; Alexandrova et al., 2008; Gardel et al., 2008).

Mainly in the rear part of the cell, the interplay of actin movement and dynamic substrate anchorage leads to the formation of parallel actin bundles by a barely understood mechanism (Svitkina et al., 1997; Hotulainen and Lappalainen, 2006; Vicente-Manzanares et al., 2008). These thick actin bundles apply large traction force to the substrate through terminally located FAs (Oliver et al., 1995; Möhl et al., 2009). This mechanical load, in turn, can either promote the growth of FAs (Balaban et al., 2001; Galbraith et al., 2002) and the recruitment of zyxin (Colombelli et al., 2009), or lead to rupture of bonds and subsequent FA disassembly (Paul et al., 2008). Thus, the organization of the actin network is highly dependent on the distribution of FAs and vice versa. These various interactions produce complex and unpredictable behavior, such as randomly occurring protrusions and retractions of the leading edge. Within the cell, FA dynamics are spatially and temporally controlled through a number of signaling pathways. The FAK-c-Src complex, for example, activates FA turnover by phosphorylating vinculin or paxillin (Bellis et al., 1995; Zhang et al., 2004; Wagner et al., 2008; Broussard et al., 2008). The regulation of FAK activity, in turn, is interconnected with the regulation of actin ploymerization through Rho family kinases (Vicente-Manzanares et al., 2005), which allows the coordination of actin and FA dynamics.

The interplay between actin and FA dynamics generates an effective traction force pattern propelling the cell forward. To date, it is still unclear how regulated FA assembly and disassembly contribute to this pattern of traction forces. Computational simulations are a promising way of gaining a deeper insight into the interplay between cytoskeleton and FA dynamics and the resulting tractions (Mogilner and Verzi, 2003; Kruse et al., 2006; Kuusela and Alt, 2009). However, theoretical modeling requires structural information and validation based on experimental data. Recently, numerous experiments were devoted to the careful quantification of actin flow and traction forces in migrating cells (Beningo et al., 2001; Vallotton et al., 
2005; Hu et al., 2007; Schaub et al., 2007; Gardel et al., 2008; Möhl et al., 2009; Rubinstein et al., 2009; Fournier et al., 2010). Although FA turnover has been qualitatively described (Anderson and Cross, 2000; Zaidel-Bar et al., 2003; Rid et al., 2005), quantitative measurements have not yet been published. Owing to this lack of experimentally obtained parameter values of FA dynamics, the recent cell migration model of Rubinstein et al. is restricted to actin-myosin dynamics and incorporates only a static FA distribution (Rubinstein et al., 2009).

The predominant problem in collecting reliable quantitative data on migrating cells is the high variability in all parameters such as cell shape, protein localization or traction forces. This results in extremely heterogeneous data and therefore severely hampers temporal analyses of individual cells as well as comparisons between these cells. For example morphology and cytoskeletal organization of the cell are constantly remodeled by, amongst others, iterative lamellipodial protrusions and retractions. These events are rather unpredictable in terms of time and location and sometimes involve the spontaneous repolarization of the whole cell (Verkhovsky et al., 1999; Sambeth and Baumgaertner, 2001; Yam et al., 2007). As a result, the cell performs a random walk by constantly changing its migration direction, shape and cytoskeletal organization. Because of this dynamic behaviour, the cytoskeletal organization varies immensely with time and between individual cells. Therefore, it is questionable to describe the general organization of, for example, actin or FAs in these cells on the basis of single microscopic snapshots. Instead, averaging microscopic data over time and/or various cells is highly desirable to obtain reliable generalized quantitative results on which firm conclusions on the basic principles of cell migration could be based.

To achieve this goal, we quantified the FA dynamics, actin flow and traction forces of migrating keratinocytes in separate experiments and developed a well-defined method to map these heterogeneous data sets into a normalized coordinate system where the cell shape is restricted to a unit circle. Using this approach, we aimed for the following: first, to be able to average over both time and populations, and to thus reliably extract the general behaviour of the system and its inherent variations with high spatial resolution; second, to precisely quantify the response of FA parameters to genetic perturbations; and third, to analyze spatial patterns from different types of measurement, namely adhesion dynamics, actin flow and traction forces, in direct context to each other.

\section{Results}

Data acquired using microscopy of migrating cells can be suitably mapped into a normalized cell coordinate system Particularly in the case of migrating cells, unpredictable changes in cell shape and position lead to highly heterogeneous microscopy data and impede an objective analysis by standard statistical methods such as averaging or cross correlation.

To overcome this barrier we developed a new method to align micrographs of motile cells by transforming them into a standard shape (Fig. 1 and Materials and Methods), and subsequently averaging over time and populations. Average distributions of actin filaments and FAs were determined as follows.

Keratinocytes were transfected with either GFP-actin or the FA marker GFP-vinculin and the dynamics of actin filaments and FAs were imaged in time-lapse sequences during cell migration (Fig. 1A,E; supplementary material Movie 1, left). To align the images, all frames of a time series were transferred to a cellular coordinate system with the cell center positioned at the systems origin and the direction of migration along the $y$-axis (Fig. 1B,F). For each time point the cell was re-centered and reoriented thus giving the impression of running on a treadmill. By smoothing the orientation of the cell over 5 minutes, potential noise caused by transient lamellipodial dynamics was strongly reduced (supplementary material Movie 1, middle).

Although individual cell shapes varied over time, the calculated average shape was almost circular (detailed analysis in supplementary material Fig. S1). To compensate for cell shape variations, we applied a second image transformation to the already shifted and rotated images. Here, the cell was normalized to a circle by applying a local morphing algorithm (Fig. 1C,G; supplementary material Movie 1, right; see Materials and Methods for details) to enable meaningful averaging and comparison.

\section{Focal adhesions and actin filaments show a polarized distribution with respect to the direction of migration}

Averaging of transformed GFP-vinculin and GFP-actin fluorescence micrographs revealed a polarized distribution of FAs and actin filaments in migrating cells. As illustrated in the averaged vinculin image, FAs were mainly located at the cell periphery, forming an adhesion belt along the rim of the cell. However, close to the rearmost point of the cell, the vinculin density was considerably lower indicating a local interruption of the adhesion belt (Fig. 1D). This anisotropic FA distribution with a pronounced depression at the rear proves explicitly that keratinocytes move towards regions of higher adhesion density.

The average actin signal was relatively low at the periphery but increased with increasing distance from the cell edge (Fig. 1H). However, closer to the cell center, the actin intensity decreased again and finally reached a minimum slightly rearward from the central point, where the nucleus is typically located. Directly behind the nucleus actin filaments accumulated, forming a narrow band of high fluorescence intensity. Here, the actin cytoskeleton is preferentially organized into thick parallel bundles, forming contractile structures in combination with myosin II (Hotulainen and Lappalainen, 2006; VicenteManzanares et al., 2008; Svitkina et al., 1997). In front of the nucleus the actin signal was also higher but more spread out compared with the narrow band at the rear end. This expanded frontal region corresponds most likely to lamellae regions of the cell where actin filaments are organized in dense criss-cross patterns and partially form transversal parallel bundles (Svitkina et al., 1997; Hotulainen and Lappalainen, 2006).

Comparisons of actin and FA distribution revealed a compressed actin network in regions of low adhesion (narrow band at the rear) and a more spread out one in regions of high adhesion density (lamellum region).

\section{Distinct zones of focal adhesion dynamics correlate to the migration direction}

To achieve deeper insight into the interplay of contractile and adhesive components during cell movement, we investigated how the polarized distribution of actin filaments and FAs in migrating cells is established. Therefore, we quantified growth dynamics of FAs and transformed the data using the normalized cellular coordinate system. 

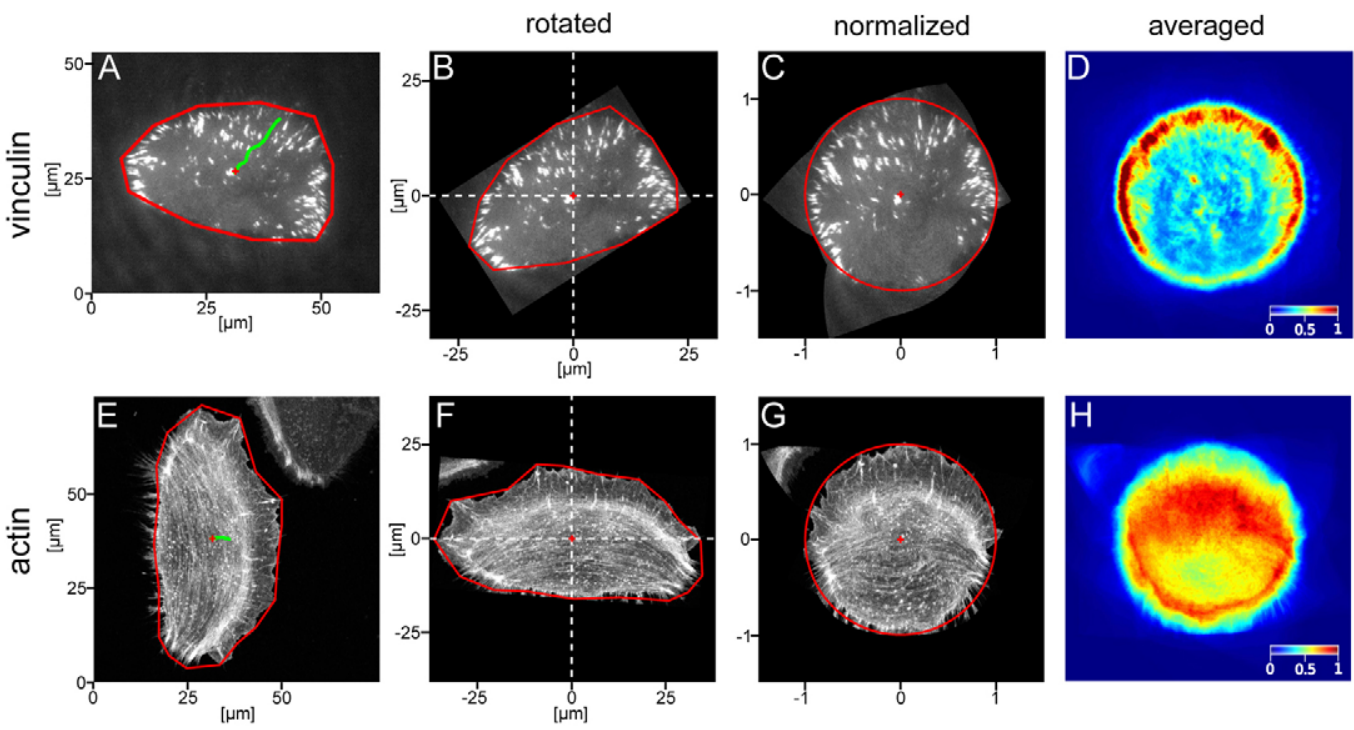

Fig. 1. Transformation of images to a normalized cellular coordinate system for averaging fluorescent signals in migrating cells. Upper row: GFPvinculin-labeled cells (see also supplementary material Movie 1). Lower row: GFP-actin-labeled cells. (A,E) Unprocessed images of migrating cells from timelapse series. Cell outline and center are marked in red. The migration trajectory, giving the direction of cell movement for every frame of the time series is denoted in green; length of time series: 2100 seconds (A), 200 seconds (E). (B,F) Images A and E transformed to the cellular coordinate system. The cell center was moved to the origin of the coordinate system in the middle of the image. Additionally, the migration direction was adjusted parallel to the $y$-axis by rotating the image. $(\mathbf{C}, \mathbf{G})$ Cells from images B and F were normalized to a circle (red line, see text for details). (D,H) Vinculin and actin distribution of migrating cells averaged in the normalized cellular coordinate system. Fluorescence was averaged by applying the transformation to all frames of several time-lapse sequences (vinculin: eight cells, 294 minutes total observation time; actin: seven cells, 23 minutes total observation time). Normalized fluorescence intensity (range from 0 to 1 , arbitrary units) is given in pseudocolors (see scale).

An image segmentation algorithm was applied to automatically track FAs in time-lapse sequences of migrating keratinocytes transfected with GFP-vinculin (Fig. 2A, see Materials and Methods for detailed description). Area profiles of individual FAs were then extracted (Fig. 2B) and used to calculate the growth-rate and age of every single FA for every time step, $t_{\mathrm{i}}$ (Fig. 2C). The shape of each FA was quantified by the aspect ratio of ellipses fitted to the detected FA patches. Additionally, continuous tracking of FA positions allowed calculation of sliding velocities. Finally, the positions were transformed to the normalized cell coordinate system to enable averaging of the data over time and over several cells.

Median values of all extracted FA parameters are shown in Fig. 2E in normalized cell coordinates. All patterns are clearly polarized (statistical spread analyzed in supplementary material Fig. S2) which confirms the direct relation between migration direction and FA dynamics described earlier by qualitative observations (Anderson and Cross, 2000). The map of FA areas (Fig. 2E, area) closely corresponds to the averaged vinculin distribution shown in Fig. 1D. This map reflects the asymmetric adhesion belt along the cell edge. Larger adhesions are located at the leading edge and at the flanks (lateral sides). The largest adhesions are found at the flanks whereas those at the rear are mostly smaller. Fig. 2D also shows FA areas that have been averaged over time and multiple cells, but here the data are only transformed to the cell coordinate system and not normalized to a circular shape. The pattern is very similar to the circular one demonstrating that the characteristic polarized distribution is prominent even without shape normalization. However, the pattern looks blurred compared with the distribution in normalized cell coordinates. This corroborates our choice of the normalized coordinate system.
According to the aspect ratio analysis, long and narrow adhesions (high aspect ratios) were predominantly found at the front, whereas large FAs at the sides are more circular (Fig. 2E, length aspect ratio). The growth rate analysis revealed well-defined zones of FA assembly and disassembly. Assembling FAs were exclusively found at the leading edge (Fig. 2E, growth rate), which is also the zone of pronounced FA nucleation (Fig. 2E, nucleation rate). This shows that the cell moves on average in the direction of newly formed and further growing FAs. Directly behind the leading edge, there is a sharp change from assembling to disassembling FAs (negative growth rate). This suggests the disassembly of a substantial number of the newly formed FAs that have departed from the leading edge. The same observation was reported for fish keratocytes (Anderson and Cross, 2000). However, FA stabilization (growth rate near zero) was observed only in the lateral parts of the cell, which could explain the increased FA size in these regions. Although the size of the FAs in the center was stable, the whole rear part was dominated by FA disassembly. The youngest FAs were predominantly located at the edges of the cell (Fig. 2E, age), with the FAs becoming older with increasing distance from the cell edge. The oldest adhesions, which were found to have a lifetime of $\sim 20$ minutes, were mostly present in the zones of FA disassembly at the front and the rear.

FA sliding was mainly observed in the disassembly zones at the rear (Fig. 2E, sliding velocity), where the adhesions moved inward with a speed of $\sim 0.1 \mu \mathrm{m} /$ minute. This result is consistent with earlier studies where stable 'towing' adhesions at the front, and forward sliding trailing adhesions were described (Anderson and Cross, 2000; Rid et al., 2005; Ballestrem et al., 2001).

Taken together, these results disclose a distinctive and dynamic pattern of FAs that is directly related to the direction 
A

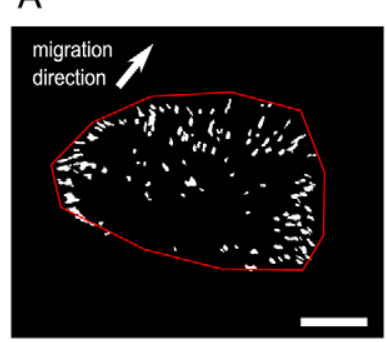

B

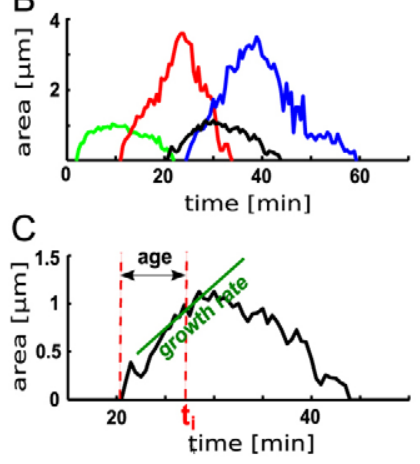

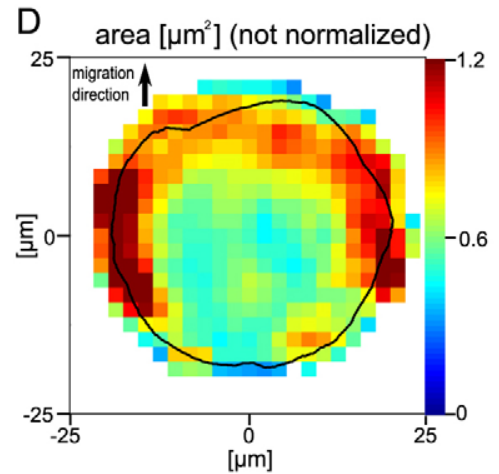

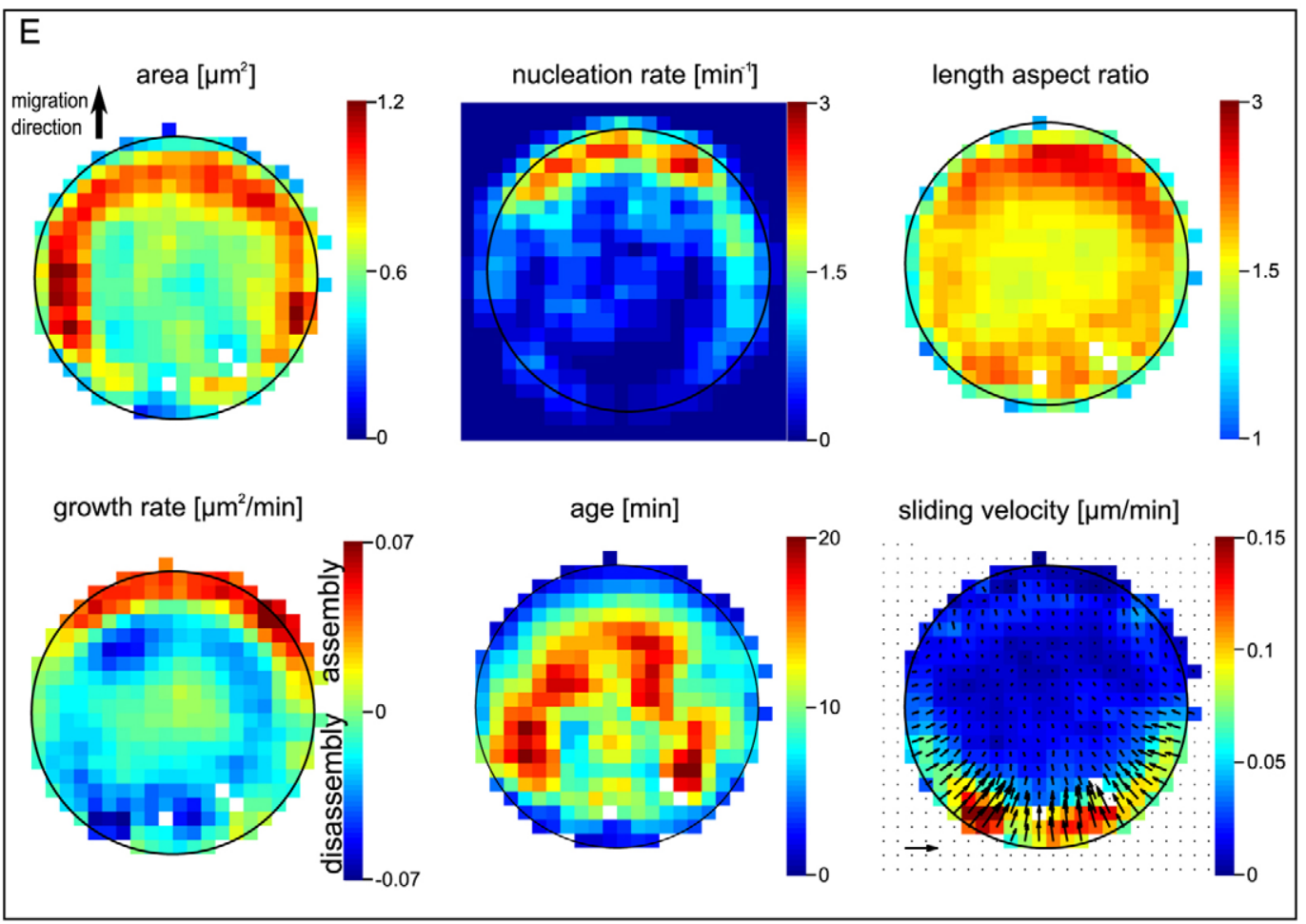

Fig. 2. Quantification of FA properties in migrating cells. All data were obtained from GFP-vinculin-transfected cells. (A) FAs (white) automatically detected from the fluorescent image shown in Fig. 1A. Cell shape is outlined in red. Scale bar: $10 \mu \mathrm{m}$. (B) Four typical area profiles of FAs. (C) Magnification of one area profile from B (black line). Additional parameters, such as growth rate and age were calculated for every time step $t_{\mathrm{i}}$. (D) Map of averaged FA areas. Data were transferred to the cellular coordinate system (see Fig. 1B) but not normalized to a circle. Data from eight cells, 294 minutes total observation time. (E) Maps of averaged FA parameters in the normalized cellular coordinate system (see Fig. 1C). In white regions (maps for area, aspect ratio, growth rate, age and sliding velocity) the number of data points is below the threshold, $n_{\min }=25$. Only the position but not the direction of sliding velocity vectors was normalized to the circular shape. Velocity vector scale: $0.15 \mu \mathrm{m} /$ minute. Data from eight cells, 294 minutes total observation time. Corresponding statistical spread is given in supplementary material Fig. S2.

of migration. FAs are mainly assembled at the cell front and mature into stable and large adhesions at the sides. Disassembly of FAs, however, occurs in the front and rear parts of the cell. Furthermore, disassembly at the rear is accompanied by fast forward sliding.

\section{Centripetal actin flow negatively correlates with focal adhesion density}

Myosin-dependent movement of actin filaments is thought to be the result of ineffective substrate coupling (Rubinstein et al., 2009; Hu et al., 2007). This well-established theory is supported by the finding that actin flow is strongly reduced above FA sites, which, in fact, act as substrate anchors for actin filaments
(Alexandrova et al., 2008; $\mathrm{Hu}$ et al., 2007). To investigate the general relationship between the global patterns of actin flow and substrate adhesion in migrating cells, we imaged GFP-actin in proximity to the substrate by TIRF microscopy and quantified actin flow by time-integrated cross correlation ( $\mathrm{Ji}$ and Danuser, 2005). The flow data were subsequently transformed to the normalized cell coordinate system. This enabled us to average over many cells and, most importantly, to directly compare FA dynamics with actin flow patterns.

As shown for FA dynamics, the actin flow was also clearly related to the direction of migration, with slow flow at the cell front and fast flow at the rear (Fig. 3A,B, single cell and averaged, respectively). Actin filaments moved generally towards 

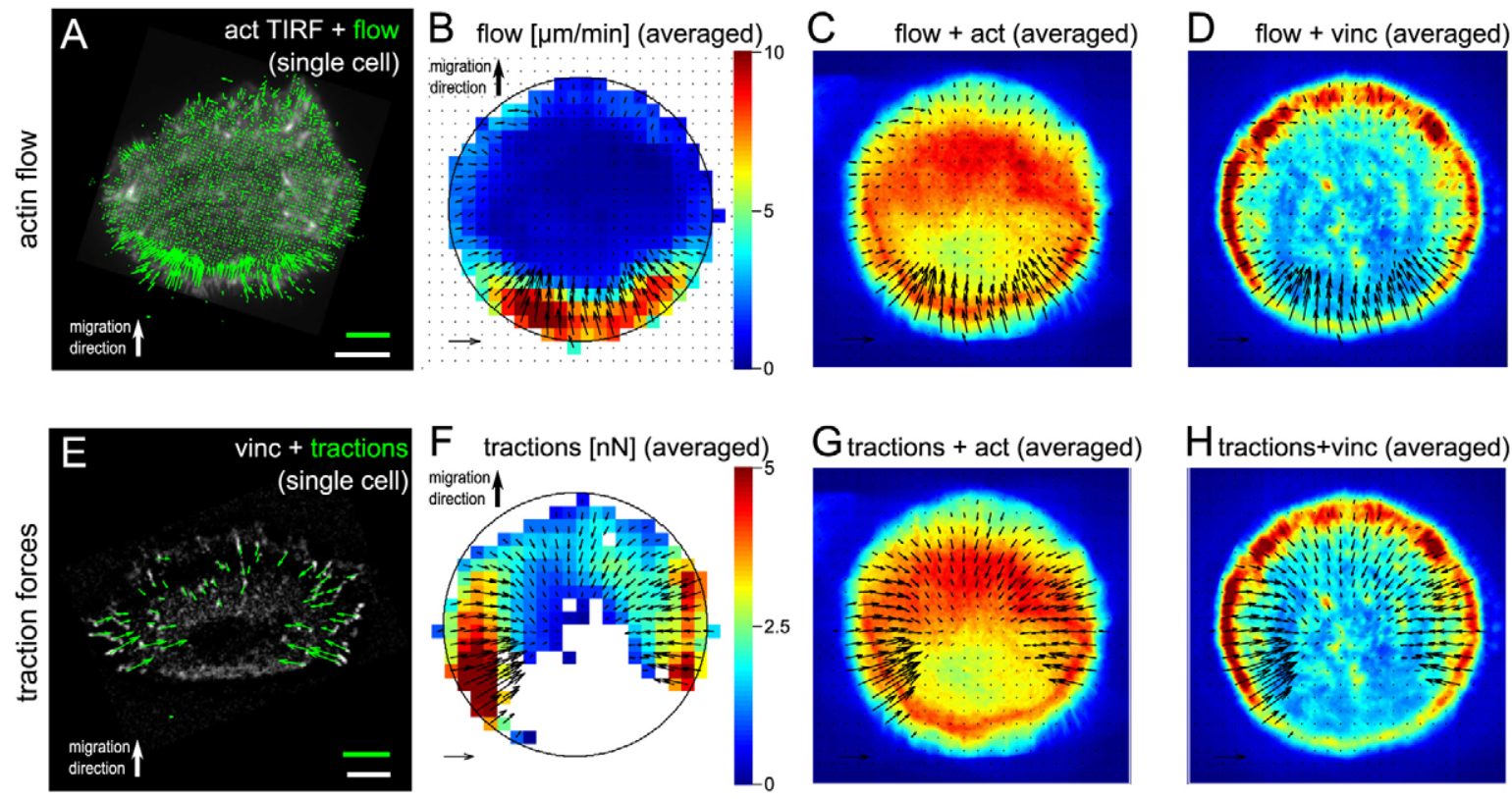

Fig. 3. Actin flow and traction forces in migrating cells. (A) Map of actin flow in a migrating keratinocyte. Flow was quantified from movies taken of GFPactin-transfected cells using TIRF microscopy over 80 seconds. The actin TIRF image is displayed in grayscale, green arrows represent flow vectors. Flow scale (green): $10 \mu \mathrm{m} /$ minute. Length scale (white): $10 \mu \mathrm{m}$. (B) Averaged actin flow shown in the normalized cell coordinate system ( $n=9$ cells). In white regions the number of data points is below the threshold, $n_{\min }=20$. Only the position but not the direction of velocity vectors was normalized to the circular shape. Scale (black arrow): $10 \mu \mathrm{m} /$ minute. (C,D) Overlay of actin flow vectors from B and averaged fluorescence images of actin (act) and vinculin (vinc; also shown in Fig. 1D and H). (E) Traction forces in a migrating keratinocyte. Fluorescence image of vinculin, showing FAs in grayscale. Force vectors for every adhesion are overlaid in green. Force scale (green): $5 \mathrm{nN}$. Length scale (white): $10 \mu \mathrm{m}$. (F) Averaged traction forces transformed to the normalized cell coordinate system ( $n=3$ cells, overall observation time: 159 minutes). In white regions the number of data points is below the threshold, $n_{\min }=5$. Only the position but not the direction of force vectors was normalized to a circle. (G,H) Overlay of force vectors from $\mathrm{F}$ and averaged fluorescence images of actin and vinculin (see $\mathrm{C}$ and $\mathrm{D}$ ).

the cell center, which illustrates nicely the contractile behaviour of the whole network. In the cell periphery, the flow was clearly complementary to the presence of FAs (Fig. 3D, vinculin distribution also shown in Fig. 1D). Very low retrograde flow (below $3 \mu \mathrm{m} /$ minute) was observed in the frontal adhesion belt. By contrast, the non-adhesive rear region was characterized by high anterograde flow speeds of up to $10 \mu \mathrm{m} /$ minute, which is consistent with earlier studies (Schaub et al., 2007). Together, these results confirm the deceleration of actin by FAs and indicate that the asymmetric actin flow pattern in migrating cells is a consequence of the polarized FA distribution.

Besides polymerization, the actin flow pattern determines the distribution of actin filaments in the cell. This relationship is illustrated by the overlay of actin fluorescence intensity and flow (Fig. 3C, actin distribution also shown in Fig. 1H). The fast anterograde actin flow at the rear clearly points to the narrow band of high actin intensity just behind the nucleus. This indicates a compression of the rear actin network caused by its fast inward flow. By contrast, the development of a more expanded actin network at the front might be the consequence of the slow inward actin flow in this region.

\section{Highest traction forces occur in zones of large and stable focal adhesions}

The application of traction forces to the substrate is the result of the force-producing actin-myosin network interacting with forcetransmitting FAs. In this way, cellular tractions can be considered as a function of both actin flow and FA turnover. To obtain a deeper insight into this interdependence, we performed traction force measurements on cells migrating on elastic substrates. The obtained forces for single adhesions were transformed to the normalized cellular coordinate system and averaged as described above. All tractions point centripetally, similar to the direction of actin flow (Fig. 3E,F single cell and averaged, respectively). Although equally oriented, the magnitudes of traction forces and actin flow show pronouncedly disparate patterns. Because traction forces depend on the presence of FAs (Fig. $3 \mathrm{H}$ ), most tractions occur in the region of the adhesion belt. The strongest tractions are found in regions of large and stable FAs, i.e. in the lateral parts of the cell where actin flow is slow (see also Fig. 2E, area and growth rate). The frontal area, which has an expanded actin network and contains a large number of small FAs, is dominated by slow flow and relatively low, rearward directed tractions. No tractions but fast actin flow were found in the FA disassembly zone at the rear (Fig. 3G,H). Besides at the cell front where both actin flow and traction forces are low, these two quantities are inversely related. The reason for this effect is the attachment of actin filaments to FAs, which on the one hand transmits force and on the other hand immobilizes actin.

In summary, highest tractions appear at sites of strong adhesion and slow actin movement. Together with previously published results (Hu et al., 2007; Alexandrova et al., 2008; Gardel et al., 2008; Fournier et al., 2010), these findings support the theory that the tension within the actin network is most 
effectively transduced at sites of strong substrate coupling, whereas in zones of weak coupling, tension leads to a contraction and thus inwards movement of the network.

\section{The nucleation of new focal adhesion sites depends on vinculin phosphorylation}

A recent study on the role of phosphorylation in the kinetic exchange of proteins between FAs and cytosol (Möhl et al., 2009) showed that inhibition of phosphorylation at a specific tyrosine residue of vinculin (Y1065) substantially reduced this molecule's exchange dynamics in nascent FAs at the cell front. To find out if this small perturbation also affects overall FA turnover within the whole migration process, we quantified and mapped FA dynamics in migrating cells, as described before, after inhibiting vinculin phosphorylation at Y1065 (Fig. 4).

By transforming the data into the normalized cell coordinate system and applying statistical analysis, we could quantify with high accuracy the changes in FA characteristics upon partial inhibition of vinculin phosphorylation. Normalized cross correlation between FA property maps of phosphorylationinhibited (Y1065F) and unaffected (Vin- $P$ ) cells revealed that the spatial patterning of all FA parameters was not drastically influenced by vinculin phosphorylation. The normalized cross correlation coefficients were all well above 0.6 (area: 0.67; nucleation rate 0.88 ; length aspect ratio: 0.66 ; growth rate: 0.66 ; age: 0.64 ; sliding velocity: $0.84 ; n=334$ ) demonstrating a similar spatial distribution of FA properties in Y1065F and Vin- $P$ cells. In contrast to the low effect in spatial patterning, we could detect partial effects on the magnitude of FA parameters (the normalized cross correlation coefficient is exclusively a measure for pattern equality and neglects different scaling and offset). Absolute values were investigated by subtracting the maps of Y1065F-cells from those of Vin- $P$ cells. The values in the resulting images represent the local increase (positive values) and decrease (negative values) of FA parameters upon inhibition of vinculin phosphorylation. As given in Fig. 5, these subtractions reveal a slight increase in FA area (median value: $5 \%$ ) and a considerable and statistically significant decrease of FA nucleation rate (median: 8\%), whereas the statistical significance of the FA area increase is ambiguous. Interestingly, for all other parameters the median value was close to $0 \%$, indicating no influence of vinculin phosphorylation overall.

These results show that FA dynamics can be modulated by vinculin phosphorylation in a targeted way. Although phosphorylated vinculin promoted the nucleation of new FAs, the spatial distribution, growth rate and overall lifetime of existing FAs was not affected.

\section{Discussion \\ Maps of dynamic cell-substrate interactions confirm previous observations and provide new insights into cell locomotion}

To our knowledge, we present the first study that obtained a generalized spatial distribution of FA dynamics in cell migration. Although the presented patterns of traction forces and actin flow have been observed before (Oliver et al., 1995; Schaub et al., 2007; Möhl et al., 2009), our cell shape normalization technique offers a new way of integrating such data with FA dynamics, and therefore provides detailed insights into the interplay of the contractile and adhesive machinery of the cell. We detected distinct zones of FA assembly and disassembly and could quantitatively compare them with averaged actin flow and traction force maps. These measurements revealed how
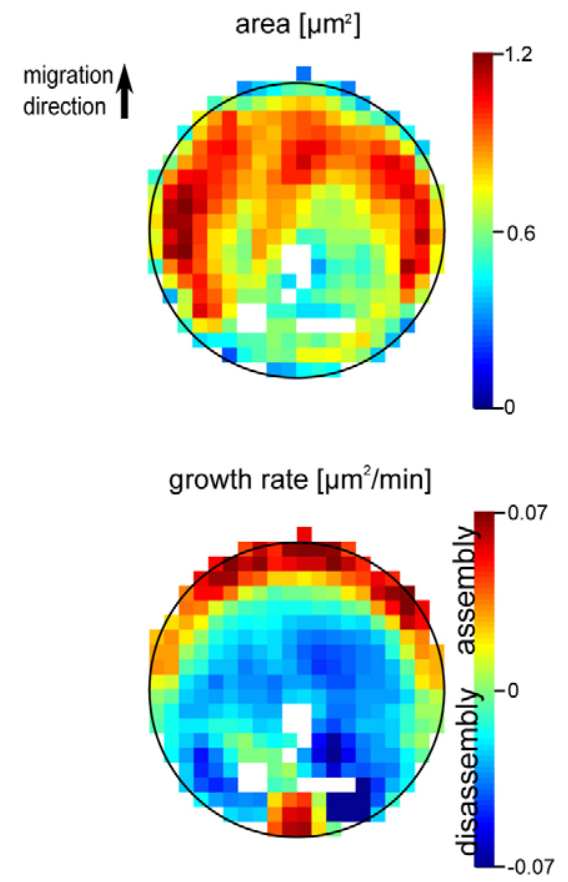
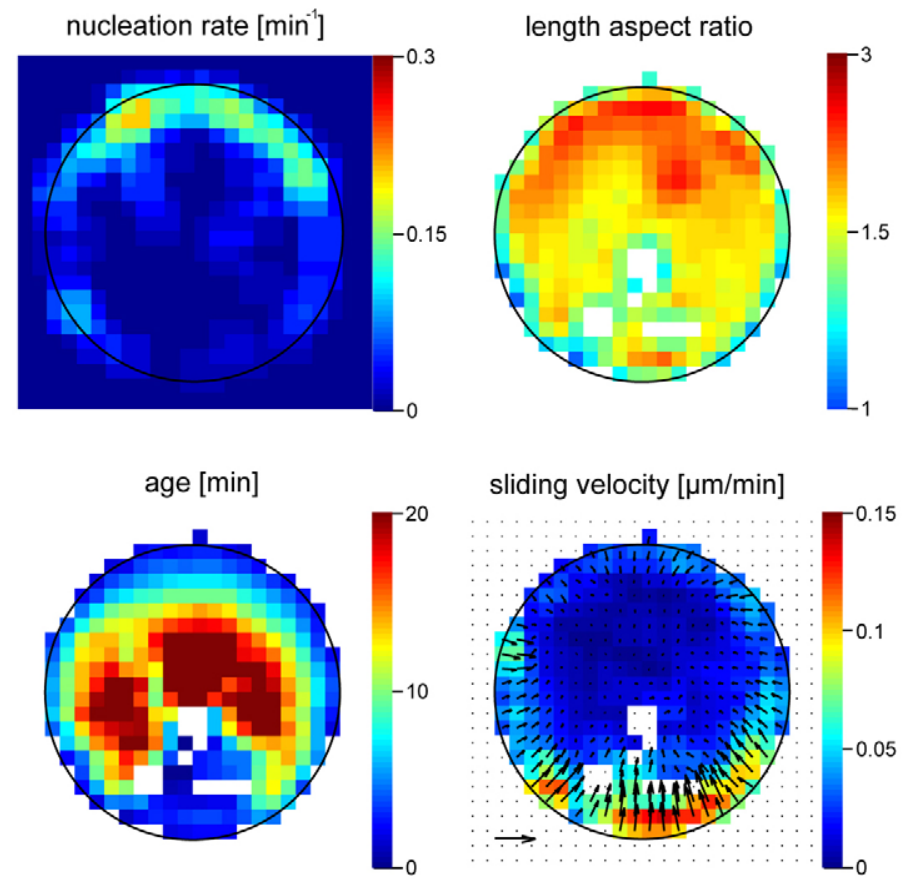

Fig. 4. Averaged FA properties in migrating cells with partially inhibited vinculin phosphorylation. All data are from GFP-vinculinY1065F-transfected

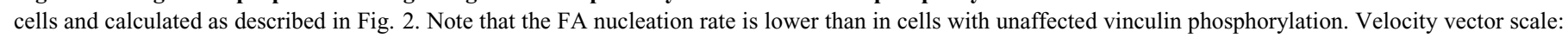
$0.15 \mu \mathrm{m} /$ minute. Data from eight cells, 350 minutes total observation time. Corresponding statistical spread is given in supplementary material Fig. S2. 


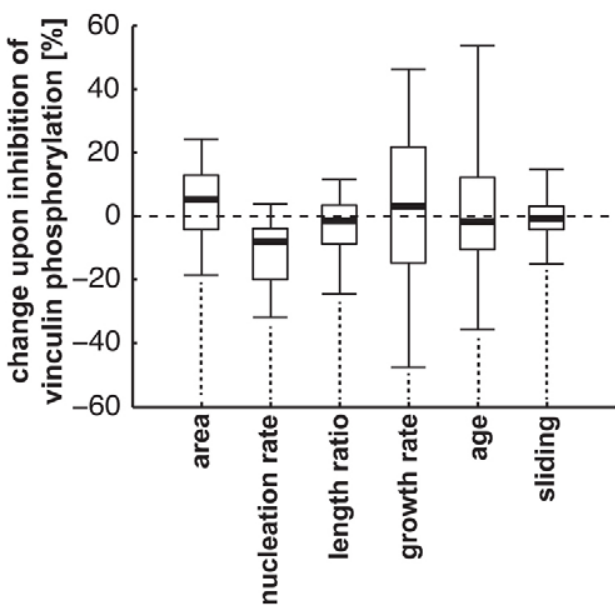

Fig. 5. Influence of vinculin phosphorylation inhibition on several FA parameters. Maps of averaged FA dynamics from cells with inhibited vinculin phosphorylation (Y1065F, Fig. 4) were quantitatively compared with maps from unaffected cells (Vin- $P$; Fig. 2D) by subtracting maps of Vin- $P$ from maps of Y1065F. Boxplots show the median and quantiles (box: 0.25 and 0.75 , whiskers: 0.05 and 0.95 ) of the resulting subtraction maps. FA area in Y1065F cells is $5 \%$ larger than Vin- $P$ cells. FA nucleation rate is $8 \%$ lower. All remaining parameters are distributed around $0 \%$, indicating that they are not influenced by vinculin phosphorylation; $n=334$ data points.

spatially distinct assembly and disassembly of FAs leads to an anisotropic actin flow pattern and to the distribution of forward propelling traction forces.
As described earlier, the spatial pattern of FA assembly and disassembly was clearly polarized in the analyzed migrating cells (Lauffenburger and Horwitz, 1996; Webb et al., 2002; Broussard et al., 2008). As a consequence of the FA growth patterns, a polarized adhesion belt was established at the cell periphery with its lowest density in the rear. In the frontal assembly zone, the adhesion belt consisted mainly of small and young FAs. Further away from the leading edge FAs were either disassembled again or grew further and maturated into large, stable and laterally located FAs (Fig. 6A). Unlike in fish fibroblasts (Rid et al., 2005), we could not detect the rearward-located assembly zone of trailing adhesions in our cells, which is probably due to the different cell type.

Substrate adhesion is known to influence the centripetal flow pattern of the actin filament network (Hu et al., 2007; Alexandrova et al., 2008). In our measurements, the inward actin flow was reduced at the front part where substrate adhesion was relatively high because of pronounced FA assembly. By contrast, the flow was maximal in the FA disassembly zone in the rear. On the basis of the FA dynamics maps presented here, this asymmetric actin flow pattern, which was also observed in fish keratocytes before (Schaub et al., 2007; Rubinstein et al., 2009), can now be explained by differential FA growth and disassembly. Interestingly, recent experiments of combined traction and actin flow measurements revealed zones of differential actin-substrate coupling, correlating to our FA dynamics maps (Fournier et al., 2010). From the results of our study we propose that the establishment of these zones is due to the spatially ordered assembly and disassembly of FAs. More precisely, the FA

\section{A: FA Turnover in a Migrating Cell}

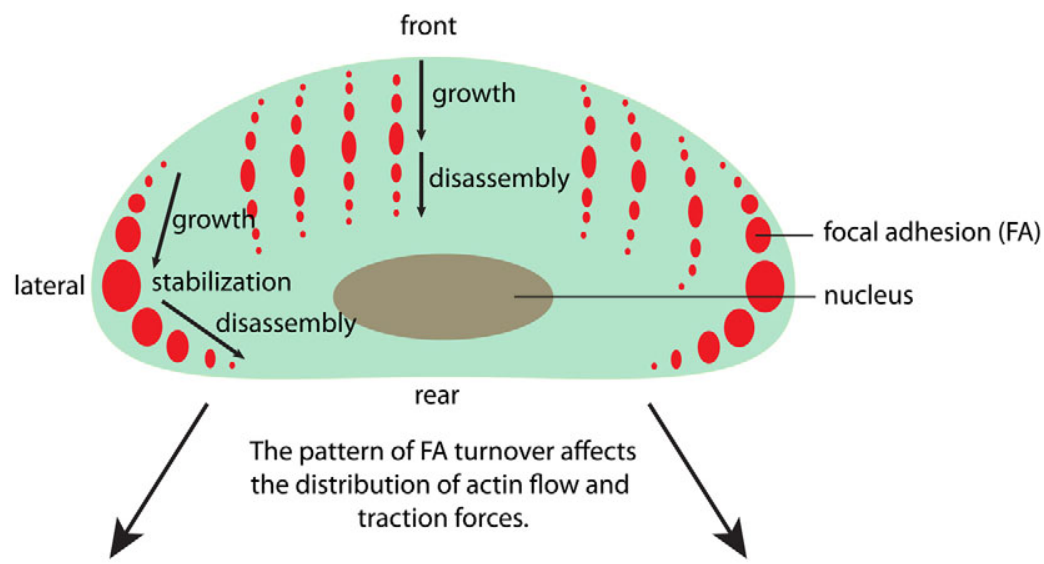

B: Actin Flow

protrusion by actin polymerization

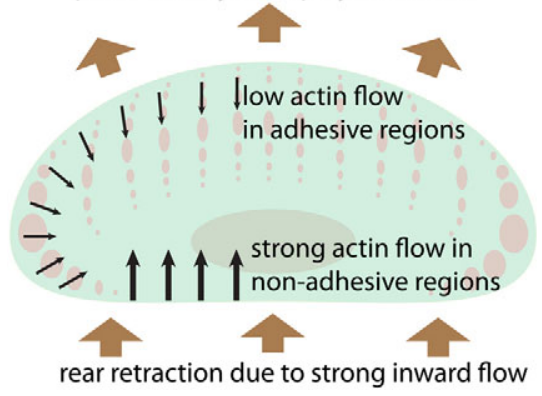

Fig. 6. Model of the interplay between FA turnover, actin flow and traction forces in migrating cells. (A) FAs (red spots) emerge at the cell front, grow and mostly disassemble when departing from the leading region. Only at the sides are FAs stabilized and they develop into large spots before they finally disassemble at the rear of the cell. As a consequence, FAs dominate in frontal and lateral regions and are almost completely missing at the rear. (B) The increased adhesiveness decelerates the actin flow (thin black arrows) in frontal and lateral regions and promotes the formation of a protruding lamella (brown arrows). By contrast, the increased actin flow (bold black arrows) at the rear promotes retraction (brown arrows). (C) The large FAs at the sides couple the actin filaments tightly to the substrate and are capable of transducing high traction forces (bold red arrows). The forces transmitted by young FAs are considerably lower (thin red arrows). No forces are transmitted in the non-adhesive region at the rear. 
assembly zone at the front corresponds to a zone of tight 'gripping', whereas so-called 'slipping' connections colocalize with the zones of large and stable FAs at the lateral flanks. The slipping connections are characterized by highest friction and force transduction, which might be a direct consequence of the increased size and stability of FAs in this area (Fig. 6C). Moreover, a zone of weak coupling with lowest friction correlates with the rear FA disassembly zone. These striking correlations indicate that substrate coupling is organized by spatially distinct growth dynamics of FAs.

The asymmetric actin flow pattern, probably caused by differential FA growth, determines, at least partially, the polarized organization of the actin cytoskeleton. Owing to the slow inward flow, the network is only slightly retracted and a lamella can be established at the front by actin polymerization (Rubinstein et al., 2009) (Fig 6B). At the rear, the network is pulled inwards with high velocity, resulting in the observed narrow actin band just behind the nucleus. Interestingly, transversally oriented thick actin bundles are often found in this region (Vicente-Manzanares et al., 2008; Möhl et al., 2009), whereas the lamellipodium is characterized by a criss-cross pattern of actin filaments (Urban et al., 2010). One can speculate about whether the strong actin flow at the rear promotes a parallel alignment of actin filaments and therefore results in formation of contractile bundles.

Our data on FA dynamics and related actin flow as well as traction force patterns agree with recent cell migration models with remarkable accuracy. Rubinstein and colleagues described the actin network as a viscous gel, which nicely described the actin flow and traction force patterns observed here and in other studies (Schaub et al., 2007; Rubinstein et al., 2009; Fournier et al., 2010). Although FA dynamics were not included in that model, the predetermined static FA distribution is similar to the asymmetric adhesion belt we observe in the present study.

As discussed above, our data suggest that controlled FA assembly and disassembly promotes the front-to-rear polarity of the cell, most apparently by formation of a lamellipodium at the front and contractile actin bundles at the rear. Therefore, signaling pathways controlling FA growth dynamics should influence cell polarization to some extent. In line with this hypothesis, the FA-regulating factor FAK, for example, has been shown to promote the reorganization of the cytoskeleton and to induce stress fiber assembly (Mitra et al., 2005).

In this context, we want to point out the power of the cell shape normalization technique to precisely quantify the effect of certain signaling events on various cellular structures and dynamics. To exemplify, we examined the effects of inhibiting vinculin phosphorylation on FA dynamics. We found that the emergence of new FAs at the front is reduced upon inhibition of vinculin phosphorylation at a specific tyrosine residue (Y1065). This indicates a critical role for phosphorylated vinculin in the nucleation of new FAs. In a recent study we observed a reduced kinetic vinculin exchange within nascent FAs upon phosphorylation inhibition (Möhl et al., 2009). Thus, enhancing binding kinetics of vinculin by phosphorylation is a possible prerequisite for FA nucleation. Interestingly, FA growth rate, lifetime and sliding velocity were not substantially influenced by vinculin phosphorylation inhibition. Therefore, FA development turns out to be a robust process where single regulatory events can precisely tune certain parameters without disturbing the whole system. This example highlights the analytical power of the cell shape normalization technique. It can be applied to numerous other processes in cell locomotion, e.g. the role of microtubules in cell polarization and FA turnover (Ezratty et al., 2005; Broussard et al., 2008) or the localization of activated regulators such as Rho or Rac (Dubash et al., 2009; Parri and Chiarugi, 2010).

\section{Significance of the cell shape normalization technique}

Although automated imaging systems provide a means of easily obtaining large data sets, there is still a lack of statistical methods for comparative single cell analysis. Averaging images of cultured cells is usually hindered by the heterogeneity of the data because the observed cells usually change location, orientation and shape over time. One solution to overcome this problem is to culture cells on micropatterned substrates, where small adhesive islands restrict the cell to a particular location and shape (Chen et al., 1997; Thery et al., 2005). However, because the cells are trapped on these islands, this approach is not suitable for studying cell migration. Instead, fish keratocytes on homogeneous substrates are often chosen as a model system for migrating cells. Their shape is relatively stable over time and can therefore be considered as a steady state system (Oliver et al., 1995; Schaub et al., 2007). In steady state, measurements at single time points theoretically reflect the average value. Hence, short-time measurements (less than 1 minute) of actin flow and traction forces in fish keratocytes have provided valuable insight into the general organization of these patterns (Vallotton et al., 2005; Schaub et al., 2007; Rubinstein et al., 2009; Fournier et al., 2010). However, for FA dynamics the relatively low number of adhesion sites is not sufficient to gain a satisfying spatial resolution on this short time scale. We calculated a mean FA density of $0.06 / \mu^{2}$, whereas actin flow was measured with a more than 10 times higher spatial resolution of 0.71 data points per $\mu \mathrm{m}^{2}$. To achieve higher statistical significance, we collected FA data over long time scales and many cells. Because we allowed the cells to move freely on a uniform substrate (as in most other studies of cell locomotion), these long-term measurements resulted in highly heterogeneous data sets. To enable subsequent averaging, all data were transformed to a standardized cell coordinate system with fixed position, orientation and shape.

A similar approach of data mapping to an object coordinate system was used in previous studies to examine the dynamics of root growth of plants (Kirchgessner et al., 2001). However, to our knowledge, a comparable technique has never been applied to mammalian cells in culture. Although we used the cell shape normalization method to study cell migration, it can, in principle, also be adapted to stationary cells. In our case, we chose direction of migration as a reference for cell orientation. In stationary cells, structural features such as the relative position of nucleus and centrosome could be taken as reference instead.

We want to point out that the presented way of mapping is suitable for extracting steady-state features. Unfortunately any temporal dynamics are removed because of the averaging process. Therefore, oscillatory behaviors and temporal correlations between parameters cannot be analyzed with this technique. For investigating temporal relationships we therefore suggest a recently developed temporal cross correlation approach, but it does lack the potential for spatial mapping (Machacek et al., 2009). 
Our approach of data alignment and averaging over long timescales has three main advantages. First, the amount of data can be extended at will by enhancing the measurement duration and/or the number of samples. Second, the general behaviour of non-steady state systems such as human keratinocytes or fibroblasts with randomly occurring protrusions and retractions can be extracted with high statistical significance. Third, data from separate experiments addressing different parameters such as FA dynamics, traction forces and actin flow can be quantitatively and reliably compared. This approach allows the fusion of independent measurements of different molecular targets to one consistent picture.

To facilitate its use, we provide a package of cell shape normalization tools including a test data set and a detailed operation manual freely available on a web server (http://www. fz-juelich.de/ics/ics-7/EN/_SharedDocs/Downloads/unitcell).

\section{Materials and Methods}

\section{Cell culture and transfection}

Normal human epidermal keratinocytes (NHEK) from neonatal foreskin were purchased from Lonza (Verviers, Belgium). Subcultures were grown in complete keratinocyte basal medium (KGM; Lonza, Cologne, Germany) at $37^{\circ} \mathrm{C}$ in $5 \% \mathrm{CO}_{2}$ Cells were harvested for subculturing or subsequent experiments using $0.025 \%$ trypsin and $0.01 \%$ EDTA in Hank's buffered salt solution (HBSS; Lonza). They were used for not more than four passages. For all experiments, 20,000-40,000 cells were plated 2-3 days before microscopic observation on glass bottom Petri dishes made in-house (thickness of glass bottom: $170 \mu \mathrm{m}$ ) or on silicone-rubbercoated coverslips (see below). All glass substrates were coated with $2.5 \mu \mathrm{g} / \mathrm{cm}^{2}$ human plasma fibronectin (BD Biosciences, San Jose, CA) for 30 minutes at room temperature before use. Silicone rubber substrates were coated with $5 \mu \mathrm{g} / \mathrm{cm}^{2}$ human plasma fibronectin for 2 hours at $37^{\circ} \mathrm{C}$

Transient transfections were performed 1 day after seeding with Transit Keratinocyte Reagent (Mirus, Madison, WI), according to the manufacturer's instructions. One of the following plasmids was used: GFP-vinculin (provided by Benjamin Geiger, Weizmann Institute of Science, Rehovot, Israel), GFPvinculinY1065F (provided by Wolfgang Goldmann, University of ErlangenNuernberg, Germany), GFP-VASP (provided by Jürgen Wehland, University of Braunschweig, Germany) and GFP-actin (pEGFP-actin; BD Biosciences, Clontech, Palo Alto, CA). For actin flow experiments, cells were double transfected with GFP-actin and DsRed-vinculin (Schäfer et al., 2010).

Cells were stimulated for migration by adding human epidermal growth factor (EGF) at a final concentration of $50 \mathrm{nM}$ (Sigma, St. Louis, MO) 1 hour before microscopy.

\section{Preparation of silicone rubber substrates}

Silicone rubber substrates [cross-linked polydimethylsiloxane (PDMS), Sylgard 184, Dow Corning GmbH, Wiesbaden, Germany] were prepared and characterized as described previously (Cesa et al., 2007). Briefly, components were mixed in a $55: 1$ base to cross-linker ratio (w/w). Five percent fluorescent beads $(0.2 \mu \mathrm{m}$ Crimson-Fluospheres, Invitrogen) were homogenized in a fraction of the still uncross-linked elastomer and poured over a silanized and microstructured silicon wafer. The layer thickness was reduced to less than $0.5 \mu \mathrm{m}$ by vigorously wiping with optical paper. Subsequently, the bead-labeled layer was overlaid with unlabeled, uncross-linked elastomer to a final thickness of approximately $100 \mu \mathrm{m}$ Cross-linking was performed at $60^{\circ} \mathrm{C}$ overnight. Calibration experiments on crosslinked silicone rubber rods (Cesa et al., 2007) from the two batches of Sylgard 184 used in this work revealed a Young's modulus of $11 \mathrm{kPa}$ and $8 \mathrm{kPa}$, respectively, and a Poisson's ratio of 0.5

\section{Microscopy}

Single migrating keratinocytes were observed by microscopy. Depending on experimental criteria, one of the following microscope setups was used. (1) An epifluorescence microscope (Observer.Z1, Carl Zeiss Microimaging, Jena, Germany) equipped for total internal reflection fluorescence (TIRF) excitation of GFP (500 mW argon ion laser; Lasos, Jena, Germany; laser-TIRF-slider, Zeiss) and wide-field illumination of DsRed (mercury arc lamp HXP 120, Osram, Augsburg, Germany). A 100x/1.46 DIC Plan-Apochromat oil objective and a CCD camera (Axiocam MRm) (both Zeiss) were used to capture micrographs. 2. A laser-scanning microscope (LSM 510 Meta, Zeiss) with a $63 \times / 1.4$ NA DIC PlanApochromat oil objective (Zeiss). The $488 \mathrm{~nm}$ line of the argon ion laser was used for GFP excitation. (3) An epifluorescence setup (Cell Observer, Zeiss) with a $40 \times / 1.3$ NA PH3 Plan-Neofluar oil objective (Zeiss). Fluorescent samples were excited with a xenon lamp (DG-4, Sutter, USA) and images were recorded with a
CCD camera (Axiocam MRm, Zeiss). All microscopes were equipped with incubators to maintain cells at $37^{\circ} \mathrm{C}$ and $5 \% \mathrm{CO}_{2}$.

FA dynamics were imaged by TIRF microscopy. For this purpose, cells were transfected with GFP-vinculin $(n=8)$ or GFP-vinculin Y1065F $(n=8)$ and recorded over periods of 13 to 86 minutes ( 2 frames per minute).

For actin flow experiments, double-transfected cells (GFP-actin, DsRedvinculin; $n=9$ ) were imaged in TIRF and epifluorescence illumination as follows. To calculate the migration direction of each cell (see below), FA dynamics were recorded in epifluorescence for 6 minutes (DsRed channel, 2 frames per minute). Directly afterwards, actin flow was measured for 80 seconds using TIRF illumination (GFP channel, 15 frames per minute). The procedure of alternating time series in either epifluorescence or TIRF mode was programmed in AxioVision 4 (Zeiss) using the SmartExperiments plug-in.

Actin distribution was imaged with the laser-scanning microscope. For this, time series of GFP-actin-transfected cells $(n=7)$ were recorded for 200 seconds each ( 6 frames per minute). One of these time series had been used in a previous work on filopodia formation (Schäfer et al., 2009).

For traction force measurements, data already published by Schäfer et al. were used (Schäfer et al., 2010). GFP-vinculin or GFP-VASP-transfected cells were imaged on bead-decorated silicone rubber substrates with the epifluorescence microscope. Movies of 37 to 85 minute duration were taken at a frame rate of 40 seconds. At every time step, fluorescent beads were imaged in red, vinculin and VASP signals as green fluorescence and the cell imaged using phase-contrast optics. Immediately after live-cell imaging, cells were detached and a reference image of the silicone rubber substrate without cell-induced deformation was taken. For this, cells were removed by trypsinization $(0.025 \%$ trypsin and $0.01 \%$ EDTA in HBSS; Lonza) for approximately 5 minutes and subsequent treatment with $20 \mathrm{mM} \mathrm{NH}_{4} \mathrm{OH}$ and $0.1 \mathrm{M} \mathrm{NaCl}$ for at least 1 hour.

Quantification of focal adhesion dynamics, actin flow and traction forces All image processing routines were implemented in Matlab 7 (The Mathworks, Natrick, MA). For analysis of FA dynamics, FAs were automatically tracked in time-lapse sequences of GFP-vinculin-transfected cells. Every frame was corrected for photobleaching by normalizing the intensity distribution to the range from zero to one and cytoplasmic background was reduced by highpass filtering as described by Zamir et al. (Zamir et al., 1999) (filter box width: $2 \mu \mathrm{m}$ ). FA regions were defined by an intensity threshold $I_{\min }=0.1$. In Fig. $3 \mathrm{~A}$, those regions are shown in white. To follow individual FAs over time, temporally and spatially overlapping regions were assigned a unique identification number using the 'union-find' algorithm (Sedgewick, 1997). All patches smaller than $0.15 \mu \mathrm{m}^{2}$ were rejected. To define FA positions, centroids were calculated for every identified patch in every image. To calculate length aspect ratios of single FAs, ellipses were fitted to all patches by a least squares method (Halir and Flusser, 1998) using the Matlab function Ellipsefit provided by Duane Hanselmann (University of Maine, Orono, ME). Growth rates were calculated as the change in FA area per time averaged over four successive time steps (2 minutes; Fig. 2C). For calculating sliding velocities, trajectories of FA positions were averaged over 2 values (1 minute)

Maps of actin flow were calculated from time series of GFP-actin TIRF images by multi-frame cross correlation. Therefore, an algorithm described by $\mathrm{Ji}$ and Danuser was implemented in Matlab (Ji and Danuser, 2005). Edge length of templates was fixed to 11 pixels $(710 \mathrm{~nm})$, and subsequent single particle tracking was omitted. A cross correlation threshold $c_{\min }=0.997$ was introduced, modifying the original procedure.

Traction force calculations were performed as described previously (Merkel et al., 2007). Briefly, positions of individual substrate-embedded microbeads were continuously recorded by video microscopy and subsequently tracked by image processing. Substrate deformation was quantified by subtracting bead positions from the corresponding positions in a reference image with no substrate deformation. Point forces were calculated for manually, defined FA positions using the deformation field and the elastic properties of the substrate as further input parameters (Schwarz et al., 2002).

\section{Image transformation and averaging}

The transformation of cell microscopy data to a standardized coordinate system was applied to fluorescence microscopy movies of FAs and actin cytoskeleton as well as to time resolved data on FA positions, actin flow and traction forces.

Transformation was performed as follows: first, the outline of a cell in each micrograph of a given movie was marked manually with $13-20$ points. The centroid of the marked area was used as the cell center (Fig. 1A,E). The smoothed trajectory of the cell center was used as the migration path of the cell. Smoothing was done by convolution with a normalized hat-like filter kernel of 5 minutes duration. To avoid edge effects the first and last 2.5 minutes of each series were rejected. For each frame the resulting trajectory reflected the average orientation of the cell within the time span defined by the filter kernel ( 5 minutes). Second, the image coordinates were recalculated by shifting the origin of the coordinate system to the respective point on the smoothed cell trajectory (which is the centroid of the 
cell) and rotating the image so that the $y$-axis aligned with the instantaneous direction of locomotion (Fig. 1B,F). Third, the shifted and aligned cell images were transformed into the normalized geometry. To this end, all points along the cell outline were transformed to polar coordinates, i.e. for each polar angle, $\varphi$, the distance between cell center and edge, $r_{\mathrm{e}}(\varphi)$, was determined. To process an entire micrograph, the shifted and aligned coordinates were expressed as polar coordinates. Subsequently, their radial components $r(\varphi)$ were divided by $r_{\mathrm{e}}(\varphi)$. This finally resulted in mapping the cell shape to a unit circle (Fig. 1C,G). In order to get dense results the inverse of this transformation was applied to the pixellated coordinates of the result image, yielding the interpolation positions in the original micrographs.

To assess mean protein distributions in cells, the movies of normalized fluorescence micrographs (actin or vinculin) were averaged. To average over many cells, the mean of all temporally averaged images was calculated (Fig. 1D,H). The averaging of FA dynamics, traction forces and actin flow data was performed as follows: the data were binned and averaged according to their position in the normalized coordinate system in a grid. The edge length of one grid cell equals $1 / 10$ of the radius of the unit cell. Taking the average cell area of $1158 \mu^{2}$ into account, the edge length of a grid cell is $1.192 \mu \mathrm{m}$. For averaging over several cells, the median of the respective parameter was determined for each grid cell. Finally, these maps were processed with a box-filter in a $3 \times 3$ neighborhood.

If the total number of data points in a single cell was below a threshold, $n_{\min }$ (FA dynamics: $n_{\min }=25$; actin flow: $n_{\min }=20$; traction forces: $n_{\min }=5$ ), the corresponding median value was rejected. In the pseudocolor images, these data points are shown in white. The statistical spread of the data was calculated as follows: first, the width of the data distribution for each cell within a single time series was characterized by the distance between the 0.25 and 0.75 quantile. Second, the corresponding distribution widths of multiple time series were calculated by their median.

To assess the credibility of these transformations, average cell shapes were determined (supplementary material Fig. S1). To this end, cell outlines were shifted and rotated as described above but not morphed to circles. These outlines were converted to binary marks, i.e. pixel values were one within the outline and zero outside. All masks of a time series were averaged, resulting in a mean image with smooth transition between 1 and 0 . The average cell shape was obtained by selecting all pixels with a value of 0.5 . We characterized all cell shapes by ellipses with identical normalized second central moments as the cell areas.

\section{Acknowledgements}

We thank Simone Born for constructing the DsRed-vinculin plasmid and Nico Hampe for calibrating silicone rubber substrates. We further thank Li-Kun Phng for critical reading of the manuscript and David Kirchenbüchler, Kevin Küpper, Wolfgang Alt and Martin Bock for stimulating discussions.

\section{Funding}

This work was supported by the Bundesministerium für Bildung und Forschung (BMBF) program [grant number 0315501].

\section{Supplementary material available online at}

http://jcs.biologists.org/lookup/suppl/doi:10.1242/jcs.090746/-/DC1

\section{References}

Alexandrova, A. Y., Arnold, K., Schaub, S., Vasiliev, J. M., Meister, J.-J., Bershadsky, A. D. and Verkhovsky, A. B. (2008). Comparative dynamics of retrograde actin flow and focal adhesions: formation of nascent adhesions triggers transition from fast to slow flow. PLoS ONE 3, e3234.

Anderson, K. I. and Cross, R. (2000). Contact dynamics during keratocyte motility. Curr. Biol. 10, 253-260.

Balaban, N. Q., Schwarz, U. S., Riveline, D., Goichberg, P., Tzur, G., Sabanay, I., Mahalu, D., Safran, S., Bershadsky, A., Addadi, L. et al. (2001). Force and focal adhesion assembly: a close relationship studied using elastic micropatterned substrates. Nat. Cell Biol. 3, 466-472.

Ballestrem, C., Hinz, B., Imhof, B. A. and Wehrle-Haller, B. (2001). Marching at the front and dragging behind: differential alphaVbeta3-integrin turnover regulates focal adhesion behavior. J. Cell Biol. 155, 1319-1332.

Bellis, S. L., Miller, J. T. and Turner, C. E. (1995). Characterization of tyrosine phosphorylation of paxillin in vitro by focal adhesion kinase. J. Biol. Chem. 270, $17437-17441$.

Beningo, K. A., Dembo, M., Kaverina, I., Small, J. V. and Wang, Y. (2001). Nascent focal adhesions are responsible for the generation of strong propulsive forces in migrating fibroblasts. J. Cell Biol. 153, 881-887.

Broussard, J. A., Webb, D. J. and Kaverina, I. (2008). Asymmetric focal adhesion disassembly in motile cells. Curr. Opin. Cell Biol. 20, 85-90.
Cesa, C. M., Kirchgessner, N., Mayer, D., Schwarz, U. S., Hoffmann, B. and Merkel, R. (2007). Micropatterned silicone elastomer substrates for high resolution analysis of cellular force patterns. Rev. Sci. Instrum. 78, 034301

Chen, C. S., Mrksich, M., Huang, S., Whitesides, G. M. and Ingber, D. E. (1997). Geometric control of cell life and death. Science 276, 1425-1428.

Colombelli, J., Besser, A., Kress, H., Reynaud, E. G., Girard, P., Caussinus, E., Haselmann, U., Small, J. V., Schwarz, U. S. and Stelzer, E. H. K. (2009). Mechanosensing in actin stress fibers revealed by a close correlation between force and protein localization. J. Cell Sci. 122, 1665.

Dubash, A. D., Menold, M. M., Samson, T., Boulter, E., García-Mata, R., Doughman, R. and Burridge, K. (2009). Chapter 1. Focal adhesions: new angles on an old structure. Int. Rev. Cell Mol. Biol. 277, 1-65.

Ezratty, E. J., Partridge, M. A. and Gundersen, G. G. (2005). Microtubule-induced focal adhesion disassembly is mediated by dynamin and focal adhesion kinase. Nat. Cell Biol. 7, 581-590.

Fletcher, D. A. and Mullins, R. D. (2010). Cell mechanics and the cytoskeleton. Nature $463,485-492$.

Fournier, M. F., Sauser, R., Ambrosi, D., Meister, J.-J. and Verkhovsky, A. B. (2010). Force transmission in migrating cells. J. Cell Biol. 188, 287-297.

Galbraith, C. G., Yamada, K. M. and Sheetz, M. P. (2002). The relationship between force and focal complex development. J. Cell Biol. 159, 695-705.

Gardel, M. L., Sabass, B., Ji, L., Danuser, G., Schwarz, U. S. and Waterman, C. M. (2008). Traction stress in focal adhesions correlates biphasically with actin retrograde flow speed. J. Cell Biol. 183, 999-1005.

Geiger, B., Bershadsky, A., Pankov, R. and Yamada, K. M. (2001). Transmembrane crosstalk between the extracellular matrix and the cytoskeleton. Nat. Rev. Mol. Cell Biol. 2, 793-805.

Halir, R. and Flusser, J. (1998). Numerically stable direct least squares fitting of ellipses. In Proceedings of the Sixth International Conference in Central Europe on Computer Graphics and Visualization, pp. 125-132. Pilzen, Czech Republic.

Hotulainen, P. and Lappalainen, P. (2006). Stress fibers are generated by two distinct actin assembly mechanisms in motile cells. J. Cell Biol. 173, 383-394.

Hu, K., Ji, L., Applegate, K. T., Danuser, G. and Waterman-Storer, C. M. (2007). Differential transmission of actin motion within focal adhesions. Science 315, 111115

Ji, L. and Danuser, G. (2005). Tracking quasi-stationary flow of weak fluorescent signals by adaptive multi-frame correlation. J. Microsc. 220, 150-167.

Kirchgessner, N., Spies, H., Scharr, H., and Schurr, U. (2001). Root growth and analysis in physiological coordinates. In Proceedings of the 11th international conference on image analysis and processing. 1, 589-594.

Kruse, K., Joanny, J. F., Jülicher, F. and Prost, J. (2006). Contractility and retrograde flow in lamellipodium motion. Phys. Biol. 3, 130-137.

Kuusela, E. and Alt, W. (2009). Continuum model of cell adhesion and migration. $J$. Math. Biol. 58, 135-161

Lauffenburger, D. A. and Horwitz, A. F. (1996). Cell migration: a physically integrated molecular process. Cell 84, 359-369.

Machacek, M., Hodgson, L., Welch, C., Elliott, H., Pertz, O., Nalbant, P., Abell, A., Johnson, G. L., Hahn, K. M. and Danuser, G. (2009). Coordination of Rho GTPase activities during cell protrusion. Nature 461, 99-103.

Merkel, R., Kirchgesner, N., Cesa, C. and Hoffmann, B. (2007). Cell force microscopy on elastic layers of finite thickness. Biophys. J. 93, 3314-3323.

Mitra, S. K., Hanson, D. A. and Schlaepfer, D. D. (2005). Focal adhesion kinase: in command and control of cell motility. Nat. Rev. Mol. Cell Biol. 6, 56-68.

Mogilner, A. and Verzi, D. W. (2003). A simple 1-D physical model for the crawling nematode sperm cell. J. Stat. Phys. 110, 1169-1189.

Möhl, C., Kirchgessner, N., Schäfer, C., Küpper, K., Born, S., Diez, G., Goldmann, W. H., Merkel, R. and Hoffmann, B. (2009). Becoming stable and strong: the interplay between vinculin exchange dynamics and adhesion strength during adhesion site maturation. Cell Motil. Cytoskeleton 66, 350-364.

Oliver, T., Dembo, M. and Jacobson, K. (1995). Traction forces in locomoting cells. Cell Motil. Cytoskeleton 31, 225-240.

Parri, M. and Chiarugi, P. (2010). Rac and Rho GTPases in cancer cell motility control. Cell Commun. Signal. 8, 23.

Paul, R., Heil, P., Spatz, J. P. and Schwarz, U. S. (2008). Propagation of mechanical stress through the actin cytoskeleton toward focal adhesions: model and experiment. Biophys. J. 94, 1470-1482.

Pollard, T. D. and Borisy, G. G. (2003). Cellular motility driven by assembly and disassembly of actin filaments. Cell 112, 453-465.

Rid, R., Schiefermeier, N., Grigoriev, I., Small, J. V. and Kaverina, I. (2005). The last but not the least: the origin and significance of trailing adhesions in fibroblastic cells. Cell Motil. Cytoskeleton 61, 161-171.

Rubinstein, B., Fournier, M. F., Jacobson, K., Verkhovsky, A. B. and Mogilner, A. (2009). Actin-myosin viscoelastic flow in the keratocyte lamellipod. Biophys. J. 97, 1853-1863.

Sambeth, R. and Baumgaertner, A. (2001). Autocatalytic polymerization generates persistent random walk of crawling cells. Phys. Rev. Lett. 86, 5196.

Schäfer, C., Borm, B., Born, S., Möhl, C., Eibl, E.-M. and Hoffmann, B. (2009). One step ahead: role of filopodia in adhesion formation during cell migration of keratinocytes. Exp. Cell Res. 315, 1212-1224.

Schäfer, C., Born, S., Möhl, C., Houben, S., Kirchgeßner, N., Merkel, R. and Hoffmann, B. (2010). The key feature for early migratory processes: dependence of adhesion, actin bundles, force generation and transmission on filopodia. Cell Adh. Migr. 4, 215-225. 
Schaub, S., Bohnet, S., Laurent, V. M., Meister, J.-J. and Verkhovsky, A. B. (2007) Comparative maps of motion and assembly of filamentous actin and myosin II in migrating cells. Mol. Biol. Cell 18, 3723-3732.

Schwarz, U., Balaban, N., Riveline, D., Bershadsky, A., Geiger, B. and Safran, S. (2002). Calculation of forces at focal adhesions from elastic substrate data: the effect of localized force and the need for regularization. Biophys. J. 83, 1380-1394.

Sedgewick, R. (1997). Algorithms in C, Parts 1-4: Fundamentals, Data Structures, Sorting, Searching, 3rd edn. London: Addison-Wesley Professional.

Svitkina, T. M., Verkhovsky, A. B., McQuade, K. M. and Borisy, G. G. (1997) Analysis of the actin-myosin II system in fish epidermal keratocytes: mechanism of cell body translocation. J. Cell. Biol. 139, 397-415.

Thery, M., Racine, V., Pepin, A., Piel, M., Chen, Y., Sibarita, J.-B. and Bornens, M. (2005). The extracellular matrix guides the orientation of the cell division axis. Nat. Cell Biol. 7, 947-953.

Urban, E., Jacob, S., Nemethova, M., Resch, G. P. and Small, J. V. (2010). Electron tomography reveals unbranched networks of actin filaments in lamellipodia. Nat. Cell Biol. 12, 429-435.

Vallotton, P., Danuser, G., Bohnet, S., Meister, J.-J. and Verkhovsky, A. B. (2005) Tracking retrograde flow in keratocytes: news from the Front. Mol. Biol. Cell 16, 1223-1231.

Verkhovsky, A. B., Svitkina, T. M. and Borisy, G. G. (1999). Self-polarization and directional motility of cytoplasm. Curr. Biol. 9, 11-20.

Vicente-Manzanares, M., Webb, D. J. and Horwitz, A. R. (2005). Cell migration at a glance. J. Cell Sci. 118, 4917-4919.
Vicente-Manzanares, M., Koach, M. A., Whitmore, L., Lamers, M. L. and Horwitz, A. F. (2008). Segregation and activation of myosin IIB creates a rear in migrating cells. J. Cell Biol. 183, 543-554.

Wagner, S., Storbek, C. J., Roovers, K., Chaar, Z. Y., Kolodziej, P., McKay, M. and Sabourin, L. A. (2008). FAK/src-family dependent activation of the ste20-like kinase SLK is required for microtubule-dependent focal adhesion turnover and cell migration. PLOS ONE 3, e1868.

Wang, Y.-L (2007). Flux at focal adhesions: slippage clutch, mechanical gauge, or signal depot. Sci. STKE 2007, pe10.

Webb, D. J., Parsons, J. T. and Horwitz, A. F. (2002). Adhesion assembly, disassembly and turnover in migrating cells - over and over and over again. Nat. Cell Biol. 4, E97-E100.

Yam, P. T., Wilson, C. A., Ji, L., Hebert, B., Barnhart, E. L., Dye, N. A., Wiseman, P. W., Danuser, G. and Theriot, J. A. (2007). Actin myosin network reorganization breaks symmetry at the cell rear to spontaneously initiate polarized cell motility. $J$. Cell Biol. 178, 1207-1221.

Zaidel-Bar, R., Ballestrem, C., Kam, Z. and Geiger, B. (2003). Early molecular events in the assembly of matrix adhesions at the leading edge of migrating cells. $J$. Cell Sci. 116, 4605-4613.

Zamir, E., Katz, B. Z., Aota, S., Yamada, K. M., Geiger, B. and Kam, Z. (1999). Molecular diversity of cell-matrix adhesions. J. Cell. Sci. 112, 1655-1669.

Zhang, Z., Izaguirre, G., Lin, S.-Y., Lee, H. Y., Schaefer, E. and Haimovich, B. (2004). The phosphorylation of vinculin on tyrosine residues 100 and 1065, mediated by src kinases, affects cell spreading. Mol. Biol. Cell 15, 4234-4247. 